\title{
Model-Independent Test of $T$ Violation in Neutrino Oscillations
}

\author{
Thomas Schwetz $\oplus^{1}$ and Alejandro Segarra $\odot^{2}$ \\ ${ }^{1}$ Institut für Astroteilchenphysik, Karlsruher Institut für Technologie (KIT), 76131 Karlsruhe, Germany \\ ${ }^{2}$ Institut für Theoretische Teilchenphysik, Karlsruher Institut für Technologie (KIT), 76131 Karlsruhe, Germany
}

(Received 6 July 2021; accepted 27 January 2022; published 1 March 2022)

\begin{abstract}
We propose a method to establish time reversal symmetry violation at future neutrino oscillation experiments in a largely model-independent way. We introduce a general parametrization of flavor transition probabilities that holds under weak assumptions and covers a large class of new physics scenarios. This can be used to search for the presence of $T$-odd components in the transition probabilities by comparing data at different baselines but at the same neutrino energies. We show that this test can be performed already with experiments at three different baselines and might be feasible with experiments under preparation or consideration.
\end{abstract}

DOI: 10.1103/PhysRevLett.128.091801

Introduction.-The violation of time reversal $(T)$ and charge-parity $(\mathrm{CP})$ symmetries are central topics in particle physics. CP violation (CPV) is one of the necessary conditions to generate a matter-antimatter asymmetry in the early Universe [1], and under the well-founded assumption of CPT conservation, $\mathrm{CPV}$ is equivalent to $T$ violation (TV). A particularly active field is the search for CPV in neutrino oscillations [2-4]. Unfortunately, the experimental signature is rather indirect, and it is not possible to construct model-independent $\mathrm{CP}$-asymmetric observables in neutrino oscillation experiments. This is related to the fundamental obstacle that experiments and detectors are made out of matter (and not antimatter). Moreover, the passage of the neutrino beam through earth matter introduces environmental CPV due to matter effects [5].

The standard approach to this problem is to perform a model-dependent fit to data. This involves the assumptions that neutrino production, detection, and propagation are fully understood in terms of Standard Model (SM) interactions, that neutrino mixing is unitary, and only the three SM neutrino flavors exist. In this case, oscillation physics can be parametrized in terms of a unitary $3 \times 3$ lepton-mixing matrix $[6,7]$ and two neutrino mass-squared differences. $\mathrm{CPV}$ is then described by a complex phase $\delta$ in the mixing matrix [2,8], which can be fitted against data. "Observation of CPV" is considered equivalent to establishing that $\delta$ is different from 0 and $\pi$ at a certain confidence level. Within this restricted framework, current data start to provide first indications of preferred regions for the parameter $\delta$ [9-13].

Published by the American Physical Society under the terms of the Creative Commons Attribution 4.0 International license. Further distribution of this work must maintain attribution to the author(s) and the published article's title, journal citation, and DOI. Funded by SCOAP .
Large activity is devoted to study the impact of nonstandard scenarios on the search for CPV in neutrino oscillations. Examples are nonunitary mixing [14,15], nonstandard neutrino interactions [16-18], or the presence of sterile neutrinos [19-21]. In such new physics scenarios, additional complex phases appear, which can act as new sources for $\mathrm{CP}$ and $T$ violation. Typically, one adopts a specific parameterization of new physics and again performs a parametric fit in the extended model. Our aim in this Letter is to go a step beyond such approaches and develop a largely model-independent test, covering a wide class of nonstandard scenarios. Our approach is based on fundamental principles about TV noted in the seminal paper by Cabibbo [2].

Model-independent description of flavor evolution.Here, we specify our approach to describe the neutrino survival and transition probabilities $P_{\alpha \beta}$, with $\alpha, \beta=e, \mu$, and $\tau . P_{\alpha \beta}$ is the probability for a neutrino $\nu_{\alpha}$ produced at the neutrino source to arrive as $\nu_{\beta}$ at the detector. We adopt the following assumptions:

(a) Propagation of the three SM neutrino states is described by a hermitian Hamiltonian $H(E, x)$, which depends on neutrino energy $E$ and, in general, on the matter density at the position $x$ along the neutrino path.

(b) We assume that for the experiments of interest, medium effects can be described to sufficient accuracy by a constant matter density, which is approximately the same for all considered experiments. This is a good approximation for experiments with baselines less than several $1000 \mathrm{~km}[22,23]$. In an accompanying paper [24], we show that for the baselines relevant for our test, the effect of nonconstant density is negligible.

Assumption (b) implies that the matter effect does not introduce environmental TV by itself [24-26]. Therefore, any observation of TV can be related to fundamental TV of the theory. (General discussions about TV in neutrino oscillations 
can be found, e.g., in Refs. [2,27-35].) Furthermore, the Hamiltonian becomes position independent, and we can diagonalize it as $H(E)=W \lambda W^{\dagger}$, with $W$ being a unitary matrix, and $\lambda=\left(\lambda_{i}\right)$ is a diagonal matrix of the real eigenvalues $\lambda_{1}, \lambda_{2}$, and $\lambda_{3}$ of $H$. Both $W$ and $\lambda$ depend on the neutrino energy. Note that we allow for arbitrary nonstandard matter effects. In general, $W$ and $\lambda$ will be different for neutrinos and antineutrinos.

(c) We allow for arbitrary (nonunitary) mixing of the energy eigenstates $\nu_{i}$ with the flavor states $\nu_{\alpha}$ relevant for detection and production,

$$
\left|\nu_{\alpha}\right\rangle=\sum_{i=1}^{3} N_{\alpha i}^{\mathrm{prod}, \mathrm{det}}\left|\nu_{i}\right\rangle .
$$

We make no specific assumption on the complex coefficients $N_{\alpha i}$. In particular, we do not relate them to the unitary matrix $W$; we allow them to be arbitrary (sufficiently smooth) functions of energy, and they can be different for neutrino production and detection, but we do assume that they are the same for different experiments (at the same energy).

Below we focus on the experimentally relevant $\nu_{\mu} \rightarrow \nu_{\mu}$ disappearance and $\nu_{\mu} \rightarrow \nu_{e}$ appearance channels. Under the assumptions (a), (b), and (c), the corresponding probabilities are obtained as [24]

$$
\begin{aligned}
P_{\mu \alpha}= & \left|\sum_{i=1}^{3} c_{i}^{\alpha} e^{-i \lambda_{i} L}\right|^{2} \\
= & \sum_{i}\left|c_{i}^{\alpha}\right|^{2}+2 \sum_{j<i} \operatorname{Re}\left(c_{i}^{\alpha} c_{j}^{\alpha *}\right) \cos \left(\omega_{i j} L\right) \\
& -2 \sum_{j<i} \operatorname{Im}\left(c_{i}^{\alpha} c_{j}^{\alpha *}\right) \sin \left(\omega_{i j} L\right),
\end{aligned}
$$

with $c_{i}^{\alpha} \equiv\left(N_{\alpha i}^{\mathrm{det}}\right)^{*} N_{\mu i}^{\mathrm{prod}}$ and $\omega_{i j} \equiv \lambda_{j}-\lambda_{i}$. Similar expressions are obtained in the context of nonunitary mixing, e.g., Refs. [36-38]. As usual, we have traded the time dependence in the evolution equation with the space coordinate, leading to the appearance of the baseline $L$ in the above probabilities. Therefore, $T$ reversal is formally equivalent to $L \rightarrow-L$ $[2,26]$. The first line of Eq. (3) is invariant under $T$, whereas the second line is $T$-odd. Fundamental TV can be established by proving the presence of the $L$-odd term in the probability.

For practical reasons, we will introduce one more assumption. We list it here for completeness and provide further discussion and motivation below:

(d) We impose that the oscillation frequencies $\omega_{i j}$ deviate only weakly from the ones corresponding to the standard three-flavor oscillation case.

Note that our assumptions (a), (b), and (c) are rather general and cover a large class of new physics scenarios, including nonstandard interactions in production, detection, and propagation [39], generic nonunitarity [36-38], or the presence of sterile neutrinos, as long as their associated oscillation frequencies are large compared to $\omega_{i j}$ [40].

The TV test.-The strategy we propose to probe the $L$-odd terms is to measure the oscillation probability as a function of $L$ at a fixed energy and check whether $L$-even terms are enough to describe the data or if TV is required. Under these conditions, the effective frequencies and mixings in the Hamiltonian are the same, and so the data at different baselines (but at the same energy) can be consistently combined. Notice that antineutrino data cannot be analyzed together with neutrino data, as their effective frequencies and mixings are, in general, different from the neutrino's; nevertheless, separate tests could be made for neutrino and antineutrino data.

In the absence of $\mathrm{TV}$, all $c_{i}^{\alpha}$ are real, and the data points could be described by the $L$-even part of the oscillation probability. We define $\left(c_{i}^{\alpha}\right.$ real $)$

$$
P_{\mu \alpha}^{\mathrm{even}}(L, E ; \theta)=\sum_{i}\left(c_{i}^{\alpha}\right)^{2}+2 \sum_{j<i} c_{i}^{\alpha} c_{j}^{\alpha} \cos \left(\omega_{i j} L\right) .
$$

For the two relevant channels, these probabilities depend on eight parameters, which we collectively denote by $\theta$ : six real coefficients $c_{i}^{\mu}, c_{i}^{e}(i=1,2$, and 3$)$ and two independent $\omega_{i j}$, e.g., $\omega_{21}$ and $\omega_{31}$. We assume now that the probabilities $P_{\mu \mu}$ and $P_{\mu e}$ are measured at a fixed energy at several baselines $L_{b}$. We denote the corresponding measured values by $p_{b}^{\mathrm{dis}}$ and $p_{b}^{\text {app }}$ with the uncertainties $\sigma_{b}^{\text {dis }}$ and $\sigma_{b}^{\text {app }}$, respectively. Below we are going to assume that $p_{b}^{\text {dis }}$ and $p_{b}^{\text {app }}$ correspond to the values predicted by standard three-flavor neutrino $(3 \nu)$ oscillations in matter.

We now ask the question if we can exclude the hypothesis of $T$ conservation parametrized by Eq. (4) if the data correspond to $3 \nu$ oscillations with $\mathrm{TV}$, i.e., for a $\mathrm{CP}$ phase $\delta$ different from 0 or $\pi$. To this aim, we construct the $\chi^{2}$ function,

$$
\begin{aligned}
\chi_{\text {even }}^{2}(E ; \theta)= & \sum_{b=1}^{N_{L}}\left[\frac{P_{\mu \mu}^{\text {even }}\left(L_{b}, E ; \theta\right)-p_{b}^{\mathrm{dis}}}{\sigma_{b}^{\mathrm{dis}}}\right]^{2} \\
& +\sum_{b=1}^{N_{L}}\left[\frac{P_{\mu e}^{\mathrm{even}}\left(L_{b}, E ; \theta\right)-p_{b}^{\mathrm{app}}}{\sigma_{b}^{\mathrm{app}}}\right]^{2} .
\end{aligned}
$$

The best-fit $T$-conserving model is obtained by considering $\chi_{\min }^{2}(E)=\min _{\theta}\left[\chi_{\text {even }}^{2}(E ; \theta)\right]$. We will take the value of $\chi_{\min }^{2}(E)$ as a rough indication of how strongly $T$ conservation can be excluded by data and leave a more detailed statistical analysis for future work. Considering that each baseline provides two data points (appearance and disappearance) and that the $T$-even model has eight parameters, it is clear that we need more than four experiments at different baselines. Let us note, however, that our parametrization includes so-called zero-distance effects, due to the nonunitary mixing in Eq. (1). Therefore, the near-detector(s) of long-baseline experiments provide already two data points at 
$L \approx 0$, and effectively, only more than three experiments are needed.

This requirement can even be further relaxed if we impose one additional assumption, which can be motivated by the fact that we have overwhelming evidence that the standard three-flavor scenario is approximately correct and any new physics effect can only be subleading. Therefore, we introduce assumption (d) mentioned above: We assume that the oscillation frequency $\omega_{21}$ deviates only weakly from the one corresponding to the standard $3 \nu$ case. Technically, we impose this requirement by calculating the effective mass-squared difference in matter, $\Delta \tilde{m}_{21}^{2}(E)$, assuming the standard matter effect and add the following prior to Eq. (5):

$\chi_{\text {even }}^{2}(E ; \theta) \mapsto \chi_{\text {even }}^{2}(E ; \theta)+\left[\frac{\Delta \tilde{m}_{21}^{2}(E)-2 E \omega_{21}}{\sigma_{21}}\right]^{2}$.

The frequencies $\omega_{i j}$ are determined by the effective evolution Hamiltonian, assumption (a), and are independent of the mixing in Eq. (1). A general parameterization of the Hamiltonian is provided by the nonstandard neutrino interaction scenario; see, e.g., Ref. [41]. Using the results of Ref. [41], we estimate the possible deviation to $\sigma_{21}=$ $0.1 \Delta \tilde{m}_{21}^{2}$; see the Supplemental Material [42] for more details. It turns out that the other independent frequency, $\omega_{31}$, is effectively constrained by the long-baseline data used in our fit, and therefore, it is not necessary to impose an analogous prior for it. The prior in Eq. (6) acts as an additional data point for each energy bin (note that also the prior is energy dependent). Therefore, under this additional assumption, we come to the remarkable result that our model-independent test can be performed already with three experiments at different baselines plus near detectors.

The crucial requirement, however, is sufficient overlap in neutrino energy. If experiments have overlapping energy ranges, we can combine information from different energies. However, to be completely model independent, the minimization has to be done individually for each energy since we do not want to make any assumptions about the energy dependence of the unknown new physics. This is an important difference to usual model-dependent analyses.

Realistic baselines and energies. - Let us now consider planned long-baseline accelerator experiments in order to see if such a test realistically can be carried out in the future. We consider the following experiments: the DUNE project in USA $(L=1300 \mathrm{~km})[43,44]$, T2HK in Japan $(L=295 \mathrm{~km})$ [45], with the option of a second detector in Korea, T2HKK ( $L=1100 \mathrm{~km}, 1.5^{\circ}$ off axis) [46], and a long-baseline experiment at the European Spalation Source in Sweden, ESS $\nu$ SB $(L=540 \mathrm{~km})[47,48]$.

Expected event numbers are obtained from design reports or detailed studies of the physics potential and are shown for the appearance channel in the case of $3 \nu$ oscillations and $\delta=90^{\circ}$ in Fig. 1. In practice, we will see that only the two

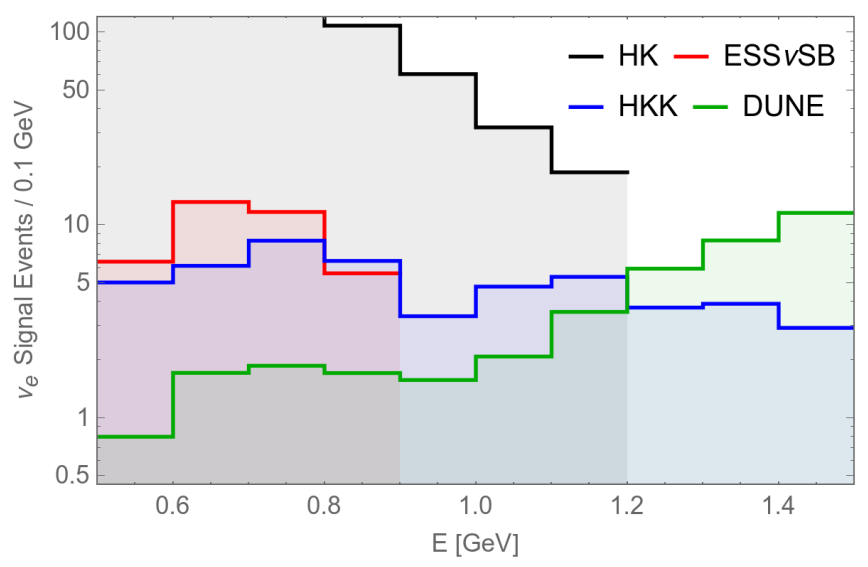

FIG. 1. Estimated number of appearance signal events at future accelerator experiments, assuming normal mass ordering and true $\delta=90^{\circ}$. Data from Refs. [49,50] (DUNE), [45] (T2HK), [46] (T2HKK), and [48] (ESS $\nu \mathrm{SB})$.

energy bins between 0.7 and $0.9 \mathrm{GeV}$ provide relevant sensitivity, as data points with sufficient statistics are needed at first and second oscillation maxima. We note that the energy spectrum from the $\mathrm{NO} \nu \mathrm{A}$ experiment [10] has no overlap with the T2K beam, and therefore, it cannot be used for this analysis. We use the information from Fig. 1 (and the corresponding data for the disappearance channel) to estimate the statistical uncertainties in Eq. (5) as $\sigma_{b r} / P^{\text {even }}\left(L_{b}, E_{r}\right)=\sqrt{S_{b r}+B_{b r}} / S_{b r}$ at baseline $b$ and energy bin $r$. We take the background events $B_{b r}$ directly from the experimental studies and estimate the number of signal events from the $N_{b r}$ in the figure, assuming $S_{b r}=N_{b r} \times P^{\text {even }}\left(L_{b}, E_{r} ; \theta\right) / P^{3 \nu}\left(L_{b}, E_{r}\right)$. For the near detector data points, we assume the standard $P_{\alpha \beta}(L \rightarrow 0)=$ $\delta_{\alpha \beta}$ with $\sigma=0.01$.

In Fig. 2, we show the data points for the appearance and disappearance probabilities as a function of the baseline for the $0.7-0.8 \mathrm{GeV}$ energy bin. We can see that the disappearance data points essentially fix the oscillation frequency, whereas the appearance data are crucial for the TV test. The "true" oscillation probability assumed to generate the data points correspond to standard $3 \nu$ oscillations with maximal $\mathrm{TV}\left(\delta=90^{\circ}\right)$ and normal mass ordering. We find that no satisfactory $L$-even fit is possible for the $4 L$ and $3 L$ (HKK) combinations at this energy. The essential information is obtained from the relative heights of the first and second appearance oscillation peaks; see the Supplemental Material [42] for further discussion. Note that disappearance probabilities can reach values larger than one in our fit, since we do not impose unitarity in our effective parameterization of the $T$-even transitions.

In order to connect our test with experiments, one should take into account the fact that finite energy resolution effectively changes the $L$ dependence in their measurements, which will, in turn, affect the sensitivity of the TV test. We assume a given energy resolution $\Delta E$ around the central bin energy $E_{0}$ and smear the transition probability by 


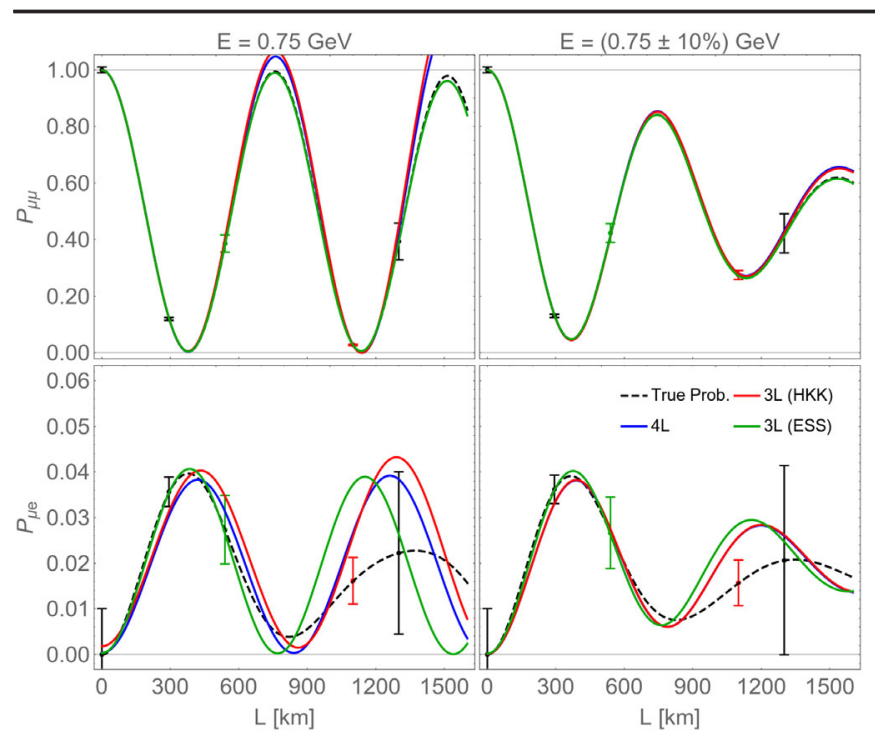

FIG. 2. Data points for the disappearance (top) and appearance (bottom) channels at the baselines of DUNE, T2HK, T2HKK, $\mathrm{ESS} \nu \mathrm{SB}$, and a near detector location for $E=0.75 \mathrm{GeV}$. Data points are generated for standard three-flavor oscillations in matter with normal mass ordering and $\delta=90^{\circ}$, and the corresponding oscillation probability is shown as black dashed. Error bars show $1 \sigma$ statistical errors. The solid curves show the best-fit model-independent $L$-even probabilities using all baselines $(4 L$, blue), DUNE + T2HK + T2HKK [3L (HKK), red], or DUNE + $\mathrm{T} 2 \mathrm{HK}+\mathrm{ESS} \nu \mathrm{SB}$ [3L (ESS), green]. Left (right) panels are without (with) the smearing due to a $10 \%$ energy resolution.

convoluting it with a Gaussian with mean $E_{0}$ and width $\Delta E$. To illustrate the effect, we assume here $\Delta E=0.1 E_{0}$. In order to perform the convolution, one must assume a certain energy dependence of the transition probability. Our assumption is that the energy dependence of the amplitudes $c_{i}^{\alpha}$ is slow enough, such that it can be neglected within an interval of few $\Delta E$. The only significant energy dependence would thus be in the oscillation phases $\omega_{i j}$. According to assumption (d) introduced above, we assume that $\omega_{31} \propto 1 / E$, as in the standard $3 \nu$ oscillation case. We have checked that our results are independent of energy smearing of $\omega_{21}$ terms. The impact of the finite energy resolution is illustrated in the right panels of Figure 2.

Our results for maximal TV are summarized in Table I, which shows the $\chi_{\min }^{2}$ values for the various energy bins for different experiment combinations, with and without including the energy smearing. We observe that $0.75 \mathrm{GeV}$ is the most relevant energy bin, whereas the one at $0.85 \mathrm{GeV}$ still provides some sensitivity. The strong impact of the energy resolution is apparent. We also find that the detector in Korea is essential, whereas both DUNE and ESS provide little sensitivity but at least one of them is needed to fix the $\omega_{i j}$ from disappearance data.

In Fig. 3, we show the summed $\chi_{\min }^{2}$ contributions from the 0.75 and $0.85 \mathrm{GeV}$ bins as a function of the value of the $3 \nu$ CP phase $\delta$ assumed to calculate the "data" to which the
TABLE I. Fit to data with the $\Delta m_{21}^{2}$ prior $\sigma_{21}=0.1$ in Eq. (6), assuming normal mass ordering and a true $\delta=90^{\circ}$. Units of $E$ are $\mathrm{GeV}$. Columns correspond to different combinations of DUNE, T2HK, T2HKK, ESS 2 SB, either without (w/o) one of them or considering all of them; the last row shows the summed total of all energy bins. The values outside (inside) the brackets show the $\min \left(\chi^{2}\right)$ without (with) smearing the data with a $10 \%$ energy resolution.

\begin{tabular}{lcccc}
\hline \hline$E$ & w/o HKK & w/o DUNE & w/o ESS & All \\
\hline 0.65 & $0.07[0.03]$ & $0.76[0.65]$ & $0.04[0.21]$ & $0.79[0.67]$ \\
0.75 & $0.04[0.04]$ & $6.95[4.78]$ & $7.92[4.82]$ & $8.60[4.86]$ \\
0.85 & $0.54[0.53]$ & $0.76[2.18]$ & $2.75[2.96]$ & $3.15[3.06]$ \\
0.95 & & & $0.42[0.98]$ & \\
Tot. & $0.65[0.60]$ & $8.46[7.60]$ & $11.13[8.97]$ & $12.54[8.59]$ \\
\hline \hline
\end{tabular}

$T$-even model is fitted. In addition to the features mentioned above, we see from Fig. 3 that the test is sensitive only to $\delta \simeq 90^{\circ}$, whereas no sensitivity appears around $270^{\circ}$. This behavior stems from the enhancement of the second oscillation maximum in the latter case (contrary to its suppression around $90^{\circ}$ ): Only when the second oscillation maximum is smaller than the first one does the $P_{\mu e}^{\text {even }}(L)$ fail to fit the data. Bins with $E>1 \mathrm{GeV}$ are not useful in the test because of the absence of measurements at both maxima. See the Supplemental Material [42] for further discussion. For illustration purpose, we show in Fig. 3 the effect doubling the event numbers in DUNE. This shows that there is significant potential to increase the sensitivity of the test by suitable optimizations. The increased sensitivity emerges from the $0.85 \mathrm{GeV}$ bin, since at this energy, the DUNE baseline is close to the second oscillation maximum.

The results for inverted mass ordering (IO) are qualitatively similar to the one from normal ordering (for IO, we

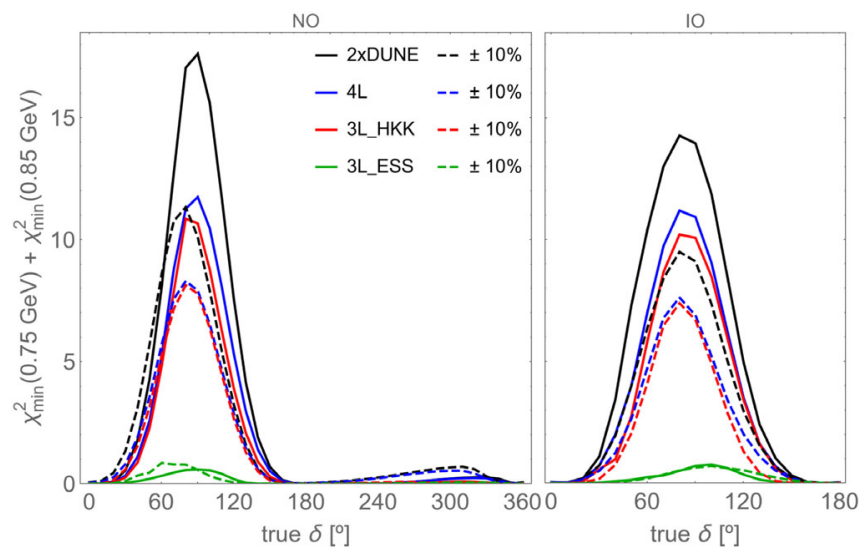

FIG. 3. $\chi_{\min }^{2}$ summed for the energy bins around 0.75 and $0.85 \mathrm{GeV}$, with perfect (solid) or $10 \%$ (dashed) energy resolution. We show the fit to all four experimental baselines (4L), DUNE + $\mathrm{T} 2 \mathrm{HK}+\mathrm{T} 2 \mathrm{HKK}[3 L(\mathrm{HKK})]$, and DUNE + T2HK+ESS $2 \mathrm{SB}$ [ $3 L$ (ESS)], as well as the effect in $4 L$ of DUNE having twice as many events (2xDUNE). Neutrino data is assumed, with normal (inverted) mass ordering for the left (right) panel. 
show only the relevant range of $\delta$ in Fig. 3). Further details on IO are given in the Supplemental Material [42]. If antineutrino data are assumed (instead of neutrino data), the result is roughly obtained for $\delta \rightarrow 2 \pi-\delta$ in Fig. 3, with highest sensitivity around $\delta \simeq 270^{\circ}$. This is to be expected since antineutrino oscillation probabilities are obtained from the neutrino ones by replacing $\delta \rightarrow-\delta$ (in addition to the sign-flip of the matter potential).

Summary.-We propose a largely model-independent test to search for $T$ violation in neutrino oscillations by comparing transition probabilities at the same energy and different baselines. The test can be done under rather general assumptions covering a wide range of new physics scenarios. Within some modest assumptions, the test can be performed already with experiments at three different baselines plus near detectors. The crucial requirements are sufficient event numbers in the neutrino energy overlap region between the experiments and good neutrino energy reconstruction [50,51]. Our estimates show that with the planned long-baseline experiments DUNE, T2HK, and T2HKK, this test can be potentially carried out. In order to cover all $T$-violating values of $\delta$, data for neutrinos and antineutrinos are necessary. We stress that a detector at the Tokai-Korea baseline is required in addition to DUNE and T2HK. Some optimization studies, especially in the lowenergy region of the DUNE and high-energy region of the T2HKK beams, may be required. The results presented here warrant more detailed sensitivity studies based on realistic experiment simulations and statistical analyses, which we leave for future work.

This project has received support from the European Union's Horizon 2020 research and innovation programme under the Marie Sklodowska-Curie Grant Agreement No. 860881-HIDDeN and from the Alexander von Humboldt Foundation.

[1] A. D. Sakharov, Violation of CP invariance, C asymmetry, and baryon asymmetry of the universe, Pis'ma Zh. Eksp. Teor. Fiz. 5, 32 (1967).

[2] N. Cabibbo, Time reversal violation in neutrino oscillation, Phys. Lett. 72B, 333 (1978).

[3] S. M. Bilenky, J. Hosek, and S. T. Petcov, On oscillations of neutrinos with Dirac and Majorana masses, Phys. Lett. 94B, 495 (1980).

[4] V. D. Barger, K. Whisnant, and R. J. N. Phillips, CP Violation in Three Neutrino Oscillations, Phys. Rev. Lett. 45, 2084 (1980).

[5] L. Wolfenstein, Neutrino oscillations in matter, Phys. Rev. D 17, 2369 (1978).

[6] B. Pontecorvo, Mesonium and anti-mesonium, Zh. Eksp. Teor. Fiz. 33, 549 (1957) [Sov. Phys. JETP 6, 429 (1957)].

[7] Z. Maki, M. Nakagawa, and S. Sakata, Remarks on the unified model of elementary particles, Prog. Theor. Phys. 28, 870 (1962).
[8] M. Kobayashi and T. Maskawa, CP violation in the renormalizable theory of weak interaction, Prog. Theor. Phys. 49, 652 (1973).

[9] K. Abe et al. (T2K Collaboration), Constraint on the matterantimatter symmetry-violating phase in neutrino oscillations, Nature (London) 580, 339 (2020); 16, 583(E) (2020).

[10] M. Acero et al. (NOvA Collaboration), First Measurement of Neutrino Oscillation Parameters Using Neutrinos and Antineutrinos by Nova, Phys. Rev. Lett. 123, 151803 (2019).

[11] I. Esteban, M. C. Gonzalez-Garcia, M. Maltoni, T. Schwetz, and A. Zhou, The fate of hints: Updated global analysis of three-flavor neutrino oscillations, J. High Energy Phys. 09 (2020) 178.

[12] P. de Salas, D. Forero, S. Gariazzo, P. Martínez-Miravé, O. Mena, C. Ternes, M. Tórtola, and J. Valle, 2020 global reassessment of the neutrino oscillation picture, J. High Energy Phys. 02 (2021) 071.

[13] F. Capozzi, E. Di Valentino, E. Lisi, A. Marrone, A. Melchiorri, and A. Palazzo, Addendum to: Global constraints on absolute neutrino masses and their ordering, Phys. Rev. D 101, 116013(A) (2020); Phys. Rev. D 95, 096014 (2017).

[14] E. Fernandez-Martinez, M. B. Gavela, J. Lopez-Pavon, and O. Yasuda, CP-violation from nonunitary leptonic mixing, Phys. Lett. B 649, 427 (2007).

[15] F. J. Escrihuela, D. V. Forero, O. G. Miranda, M. Tórtola, and J. W. F. Valle, Probing CP violation with nonunitary mixing in long-baseline neutrino oscillation experiments: DUNE as a case study, New J. Phys. 19, 093005 (2017).

[16] S.-F. Ge and A. Y. Smirnov, Nonstandard interactions and the $\mathrm{CP}$ phase measurements in neutrino oscillations at low energies, J. High Energy Phys. 10 (2016) 138.

[17] A. de Gouvêa and K. J. Kelly, Nonstandard neutrino interactions at DUNE, Nucl. Phys. B908, 318 (2016).

[18] P. B. Denton, J. Gehrlein, and R. Pestes, CP -Violating Neutrino Nonstandard Interactions in Long-BaselineAccelerator Data, Phys. Rev. Lett. 126, 051801 (2021).

[19] R. Gandhi, B. Kayser, M. Masud, and S. Prakash, The impact of sterile neutrinos on CP measurements at long baselines, J. High Energy Phys. 11 (2015) 039.

[20] A. Palazzo, 3-flavor and 4-flavor implications of the latest $\mathrm{T} 2 \mathrm{~K}$ and $\mathrm{NO} \nu \mathrm{A}$ electron (anti-)neutrino appearance results, Phys. Lett. B 757, 142 (2016).

[21] J. M. Berryman, A. de Gouvêa, K. J. Kelly, and A. Kobach, Sterile neutrino at the deep underground neutrino experiment, Phys. Rev. D 92, 073012 (2015).

[22] T. Miura, T. Shindou, E. Takasugi, and M. Yoshimura, The matter fluctuation effect to $\mathrm{T}$ violation at a neutrino factory, Phys. Rev. D 64, 073017 (2001).

[23] H. Yokomakura, K. Kimura, and A. Takamura, Overall feature of $\mathrm{CP}$ dependence for neutrino oscillation probability in arbitrary matter profile, Phys. Lett. B 544, 286 (2002).

[24] T. Schwetz and A. Segarra, companion paper, $T$ violation in nonstandard neutrino oscillation scenarios, Phys. Rev. D 105, 055001 (2022).

[25] P. I. Krastev and S. T. Petcov, Resonance amplification and t violation effects in three neutrino oscillations in the earth, Phys. Lett. B 205, 84 (1988). 
[26] E. K. Akhmedov, P. Huber, M. Lindner, and T. Ohlsson, T violation in neutrino oscillations in matter, Nucl. Phys. B608, 394 (2001).

[27] T.-K. Kuo and J. T. Pantaleone, $T$ nonconservation in three neutrino oscillations, Phys. Lett. B 198, 406 (1987).

[28] S. Toshev, Maximal $T$ violation in matter, Phys. Lett. B 226, 335 (1989).

[29] J. Arafune and J. Sato, CP and T violation test in neutrino oscillation, Phys. Rev. D 55, 1653 (1997).

[30] S. J. Parke and T. J. Weiler, Optimizing T violating effects for neutrino oscillations in matter, Phys. Lett. B 501, 106 (2001).

[31] Z.-Z. Xing, Leptonic commutators and clean T violation in neutrino oscillations, Phys. Rev. D 88, 017301 (2013).

[32] S. T. Petcov and Y.-L. Zhou, On neutrino mixing in matter and $\mathrm{CP}$ and $\mathrm{T}$ violation effects in neutrino oscillations, Phys. Lett. B 785, 95 (2018).

[33] J. Bernabéu and A. Segarra, Disentangling Genuine from Matter-Induced CP Violation in Neutrino Oscillations, Phys. Rev. Lett. 121, 211802 (2018).

[34] J. Bernabéu and A. Segarra, Signatures of the genuine and matter-induced components of the $\mathrm{CP}$ violation asymmetry in neutrino oscillations, J. High Energy Phys. 11 (2018) 063.

[35] J. Bernabéu and A. Segarra, Do T asymmetries for neutrino oscillations in uniform matter have a CP-even component?, J. High Energy Phys. 03 (2019) 103.

[36] S. Antusch, C. Biggio, E. Fernandez-Martínez, M. B. Gavela, and J. Lopez-Pavon, Unitarity of the leptonic mixing matrix, J. High Energy Phys. 10 (2006) 084.

[37] F. J. Escrihuela, D. V. Forero, O. G. Miranda, M. Tortola, and J.W.F. Valle, On the description of nonunitary neutrino mixing, Phys. Rev. D 92, 053009 (2015); 93, 119905(E) (2016).

[38] C. S. Fong, H. Minakata, and H. Nunokawa, Nonunitary evolution of neutrinos in matter and the leptonic unitarity test, J. High Energy Phys. 02 (2019) 015.

[39] A. Falkowski, M. González-Alonso, and Z. Tabrizi, Consistent QFT description of nonstandard neutrino interactions, J. High Energy Phys. 11 (2020) 048.
[40] M. Blennow, P. Coloma, E. Fernandez-Martínez, J. Hernandez-Garcia, and J. Lopez-Pavon, Non-unitarity, sterile neutrinos, and nonstandard neutrino interactions, J. High Energy Phys. 04 (2017) 153.

[41] I. Esteban, M. C. Gonzalez-Garcia, M. Maltoni, I. MartinezSoler, and J. Salvado, Updated constraints on nonstandard interactions from global analysis of oscillation data, J. High Energy Phys. 08 (2018) 180; 12 (2020) 152(E).

[42] See Supplemental Material at http://link.aps.org/ supplemental/10.1103/PhysRevLett.128.091801 for further details.

[43] B. Abi et al. (DUNE Collaboration), Deep underground neutrino experiment (Dune), far detector technical design report, volume I introduction to Dune, J. Instrum. 15, T08008 (2020).

[44] B. Abi et al. (DUNE Collaboration), Deep underground neutrino experiment (Dune), far detector technical design report, volume II: Dune physics, arXiv:2002.03005.

[45] K. Abe et al. (Hyper-Kamiokande Collaboration), HyperKamiokande design report, arXiv:1805.04163.

[46] K. Abe et al. (Hyper-Kamiokande Collaboration), Physics potentials with the second Hyper-Kamiokande detector in Korea, Prog. Theor. Exp. Phys. 2018, 063 C01 (2018).

[47] E. Baussan et al. (ESSnuSB Collaboration), A very intense neutrino super beam experiment for leptonic CP violation discovery based on the European spallation source linac, Nucl. Phys. B885, 127 (2014).

[48] M. Blennow, E. Fernandez-Martinez, T. Ota, and S. RosauroAlcaraz, Physics potential of the ESS $\nu$ SB, Eur. Phys. J. C 80, 190 (2020).

[49] R. Acciarri et al. (DUNE Collaboration), Long-baseline neutrino facility (LBNF) and deep underground neutrino experiment (DUNE): Conceptual design report, volume 2: The physics program for DUNE at LBNF, arXiv:1512.06148.

[50] V. De Romeri, E. Fernandez-Martinez, and M. Sorel, Neutrino oscillations at DUNE with improved energy reconstruction, J. High Energy Phys. 09 (2016) 030.

[51] S. S. Chatterjee, P. S. B. Dev, and P. A. N. Machado, Impact of improved energy resolution on DUNE sensitivity to neutrino nonstandard interactions, J. High Energy Phys. 08 (2021) 163. 